\title{
Effects of Charge Double Layer and Colloidal Aggregation on the Isotropic-Nematic Transition of Protein Fibers in Water
}

\author{
Raffaele Mezzenga, ${ }^{* \dagger}$ Jin-Mi Jung, ${ }^{*}$ and Jozef Adamcik ${ }^{\dagger}$ \\ ${ }^{\dagger}$ ETH Zurich, Food \& Soft Materials Science, Institute of Food, Nutrition \& Health, LFO23, \\ Schmelzbergstrasse 9, 8092 Zürich, Switzerland, and Department of Physics and Fribourg Center for \\ Nanomaterials, University of Fribourg, Ch. Musée 3, CH-1700, Fribourg, Switzerland
}

\begin{abstract}
We investigate the effects of variable linear charge density and Debye length on the mesoscopic properties of $\beta$-lactoglobulin fibers in water, by changing the $\mathrm{pH}$ and ionic strength, respectively. We determine the isotropicnematic $(\mathrm{I}-\mathrm{N})$ transition by cross-polarized microscopy and quantify by atomic force microscopy the increasing tendency of the fibers to aggregate upon raising ionic strength. We then compare experimental $\mathrm{I}-\mathrm{N}$ transitions with theoretical expected values based on Onsager theory. Unlike previous reports on lyotropic liquid crystalline behavior of protein fibers, we show that, if double layer effects and aggregation of fibers are correctly included directly in the second virial coefficient and excluded volume, Onsager theory accurately predicts the experimental $\mathrm{I}-\mathrm{N}$ transition versus $\mathrm{pH}$ and ionic strength.
\end{abstract}

\section{Introduction}

The phase behavior of nonspherical particle systems has been deeply investigated on both experimental and theoretical bases. ${ }^{1-10}$ Studies have included rod-like suspensions, such as those formed by mineral particles, ${ }^{11}$ viruses,${ }^{5,6}$ polypeptides, ${ }^{12}$ or polysaccharides; ${ }^{4,8,13}$ platelets, such as Gibbsite disks, ${ }^{1}$ and also ellipsoidal candies particles. ${ }^{14}$

*To whom correspondence should be addressed: raffaele.mezzenga@ agrl.ethz.ch.

(1) Wensink, H. H.; Lekkerkerker, H. N. W. Sedimentation and multi-phase equilibria in mixtures of platelets and ideal polymer. Europhys. Lett. 2004, 66 (1), 125-131.

(2) Vroege, G. J.; Lekkerkerker, H. N. W. Theory of phase separation for a solution of tridisperse rod-like particles. Colloids Surf, A 1997, 129-130, 405-413.

(3) Gabriel, J.-C. P.; Davidson, P. New Trends in colloidal liquid crytals based on mineral moieties. Adv. Mater. 2000, 12 (1), 9-20.

(4) Inoue, T.; Ogata, S.-I.; Kakimoto, M.; Imai, Y. Triphase equilibrium in aqueous solutions of the rodlike polysaccharide schizophyllan. Macromolecules $1984,17,1419-1420$.

(5) Huang, F.; Rotstein, R.; Fraden, S.; Kasza, K. E.; Flynn, N. T. Phase behavior and rheology of attractive rod-like particles. Soft Matter 2009, 5, 27662771.

(6) Purdy, K. R. Fraden, S. Isotropic-cholesteric phase transition of filamentous virus suspensions as a function of rod length and charge. Physical Review Letters E - Statistical, Nonliear, and Soft Matter Physics 2004, 70, 061703-1061703-8.

(7) Purdy, K. R.; Varga, S.; Galindo, A.; Jackson, G.; Fraden, S. Nematic phase transitions in mixtures of thin and thick colloidal rods. Phys. Rev. Lett. 2005, 94 , 057801-1-057801-4.

(8) Fang, Y.; Takemasa, M. Rheology of schizophyllan solutions in isotropic and anisotropic phase regions. J. Rheol. 2004, 48 (5), 1147-1166.

(9) Lee, C. F. Isotropic-nematic phase transition in amyloid fibrilization. Phys. Rev. E 2009, 80, 0319021-0319026.

(10) Onsager, L. The effects of shape on the interaction of colloidal particles. Ann. N.Y. Acad. Sci. 1949, 51, 627-659.

(11) Vroege, G. J.; Thies-Weesie, D. M. E.; Petukhov, A. V.; Lemaire, B. J.; Davidson, P. Smectic liquid-crystalline order in suspensions of highly polydisperse goethite nanorods. Adv. Mater. 2006, 18, 2565-2568.

(12) Yu, S. M.; Conticello, V. P.; Zhang, G.; Kayser, C.; Fournier, M. J.; Mason, T. L.; Tirrell, D. A. Smecting ordering in solutions and films of a rod-like polymer owing to monodispersity of chain length. Nature 1997, 389, 167-170.

(13) Yanaki, T.; Norisuye, T.; Teramoto, A. Cholesteric mesophase in aqueous solutions of a triple helical polysaccharide scleroglucan. Polym. J. 1984, 16 (2), 165-173.

(14) Donev, A.; Cisse, I.; Sachs, D.; Variano, E. A.; Stillinger, F. H.; Connelly, R.; Torquato, S.; Chaikin, P. M. Improving the density of jammed disordered packings using ellipsoids. Science 2004, 13, 990-993.
Amyloidal fibers formed by heat-denatured globular proteins have also been considered, as these objects closely resemble semiflexible charged polyelectrolytes with thick cross sections. ${ }^{15,16}$ These systems have been shown to exhibit a rich and complex liquid crystalline behavior, characterized by isotropic-nematic transitions at low volume fractions. ${ }^{15,16}$ In various attempts to rationalize their isotropic-nematic behavior, previous reports (including our own work ${ }^{15}$ ) have employed a simplified Onsager theory. For suspensions of uncharged monodisperse rigid rods of length $L$ and diameter $D$, Onsager theory predicts the onset of the nematic phase at

$$
\phi_{\mathrm{IN}}=4 \frac{D}{L}
$$

Previous descriptions of liquid crystalline properties of protein amyloid fibers ${ }^{15,16}$ have assumed that the effect of double charge layer can be accounted for by replacing directly in eq $1 D$ with $D_{\text {eff }}$, where

$$
D_{\text {eff }}=D+k^{-1}\left(\ln A+C+\ln 2-\frac{1}{2}\right)
$$

with $D_{\text {eff }}$ the effective diameter, $D$ the diameter, $k^{-1}$ the Debye length, $C$ the Euler's constant, and $A$ calculated as follows: ${ }^{17}$

$$
A=2 \pi v_{\mathrm{eff}^{2}} k^{-1} Q \exp (-k D)
$$

with $v_{\text {eff }}$ being the linear charge density and $Q$ the Bjerrum length ( $\sim 0.7 \mathrm{~nm}$ in water at room temperature).

The replacement of $D$ with $D_{\text {eff }}$ appeared to be justified by the success that this simplification has produced in the correct description of the critical percolation volume fraction of charged

(15) Jung, J.-M.; Mezzenga, R. Liquid crystalline phase behaviour of protein fibers in water: experiments versus theory. Langmuir 2010, 26 (1), 504-514.

(16) Sagis, L. M. C.; Veerman, C.; van der Linden, E. Mesoscopic properties of semiflexible amyloid fibrils. Langmuir 2004, 20, 924-927.

(17) Stroobants, A.; Lekkerkerker, H. N. W.; Odijk, Th. Effect of electrostatic interaction on the liquid crystal phase transition in solutions of rodlike polyelectrolytes. Macromolecules 1986, 19, 2232-2238. 
amyloid fibrils in bulk suspensions. ${ }^{18}$ Nonetheless, direct $D \rightarrow D_{\text {eff }}$ transformation in eq 1 for the prediction of $\mathrm{I}-\mathrm{N}$ transitions for amyloid fibers has only shown coarse agreement ${ }^{16}$ or none at all ${ }^{15}$ with experimental data. Furthermore, very specific trends arising in protein-based systems, such as fiber aggregation, have not yet been considered.

Here, we show that if the double layer charge effect is directly accounted for in the excluded volume, $V_{\text {excluded }}$, together with the effects arising from fiber aggregation, Onsager theory can be correctly applied to rationalize $\mathrm{I}-\mathrm{N}$ transition in protein amyloid fibers also. To the best of our knowledge, the model presented here is the first model that can correctly account, in a quantitative way, the $\mathrm{I}-\mathrm{N}$ transition in systems as complex as amyloid protein fibers.

\section{Experimental Section}

Materials. BioPURE- $\beta$-lactoglobulin (lot JE 003-6-922, from 23 to 05-2006) was obtained from Davisco Foods International, Inc. (Le Sueur, MN). The protein was dialyzed at $4{ }^{\circ} \mathrm{C}$, freeze-dried, redissolved in Milli-Q water at $\mathrm{pH}$ 2, and converted into fibers by heat-denaturation for $300 \mathrm{~min}$ at $90{ }^{\circ} \mathrm{C}$. The measured conversion yield of monomers into fibers of $75 \%$ was taken into account to determine the final weight or volume fraction of fibers in water. The final density of the fibers was measured to be $1.002 \mathrm{~g} / \mathrm{cm}^{3}$, which results in virtually identical weight or volume fraction values. The fibers presented here are identical to those previously described, and thus, their detailed characterization will not be repeated here. More details on fiber production and characterization are given in our previous reports. ${ }^{15,19}$ Briefly, the dimensions of the fibers after preparation at $\mathrm{pH} 2$ and $90^{\circ} \mathrm{C}$ are $4 \mathrm{~nm}$ for the average diameter, $1980 \mathrm{~nm}$ for the persistence length, and $1-20 \mu \mathrm{m}$ for the contour length.

Methods. Optical Microscopy/Cross-Polarizers. The macroscopic aspect of the samples was inspected first by the naked eye under normal light to evaluate the transparency/ turbidity and determine an eventual macroscopic phase separation. The samples were then visualized between cross-polarizers to determine presence of birefringence. Optical microscopy under white light and polarized light was also employed to confirm the macroscopic observation and to determine eventual phase boundaries among coexisting phases.

Atomic Force Microscopy. A $10 \mu \mathrm{L}$ aliquot of $\beta$-lactoglobulin fibers solution diluted to $0.1 \%$ wt at $\mathrm{pH} 2$ and a given salt concentration $(0,50$, or $100 \mathrm{mM})$ was deposited onto freshly cleaved mica, incubated for $2 \mathrm{~min}$, rinsed with Milli-Q water, and dried under nitrogen. Images were collected using a MultiModeV (Veeco Inc., Woodbury, NY, USA) operated in tapping mode in air. Images were simply flattened using the Nanoscope 8.1 software and no further image processing was carried out. The width of fibers was determined using the Nanoscope 8.1 software.

Determination of the Isotropic-Nematic Phase Transition. To investigate the effect of $\mathrm{pH}$ on the $\mathrm{I}-\mathrm{N}$ transition, solutions of $1 \% \beta$-lactoglobulin fibers at $\mathrm{pH} 2$ were prepared by dilution of the $2 \%$ stock solution with Milli-Q water adjusted to $\mathrm{pH} 2$. $\mathrm{pH}$ was then adjusted to between 1 and 4 by addition of $\mathrm{HCl}$ or $\mathrm{NaOH}$ $(1 \mathrm{M})$, and then solutions were progressively diluted with Milli-Q water adjusted to the same $\mathrm{pH}$ to investigate concentrations from $1 \%$ down to $0.1 \%$. Finally, each solution was poured in a test tube and left to rest overnight at room temperature to reach equilibrium, before observation between cross-polarizers.

To investigate the effect of ionic strength on the I-N transition, solutions of $1 \% \beta$-lactoglobulin fibers at $\mathrm{pH} 2$ were first prepared

(18) Veerman, C.; Ruis, H.; Sagis, L. M. C.; van der Linden, E. Effect of electrostatic interactions on the percolation concentration of fibrillar $\beta$-lactoglobulin gels. Biomacromolecules 2002, 3, 869-873.

(19) Jung, J.-M.; Savin, G.; Pouzot, M.; Schmitt, C.; Mezzenga, R. Structure of heat-induced $\beta$-lactoglobulin aggregates and their complexes with sodium-dodecy sulfate. Biomacromolecules 2008, 9, 2477-2486.

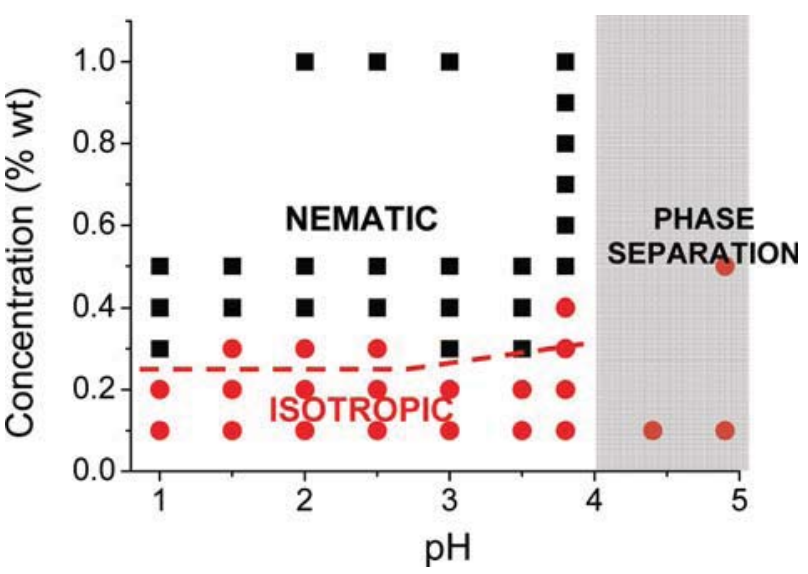

Figure 1. Critical concentration at the isotropic-nematic $(\mathrm{I}-\mathrm{N})$ transition depending on the $\mathrm{pH}$ of a suspension of $\beta$-lactoglobulin fibers in water. The red spot represents an isotropic phase; the black square represents a nematic phase. The red dashed line represents the theoretical $\mathrm{I}-\mathrm{N}$ transition predicted using eq 6 .

by dilution of the $2 \%$ stock solution. These solutions were then adjusted to the different ionic strengths from 1 to $100 \mathrm{mM}$ by addition of $\mathrm{NaCl}$. They were finally diluted using Milli-Q water adjusted to $\mathrm{pH} 2$ and having the same ionic strength, to investigate concentrations from $1 \%$ down to $0.1 \%$. The solutions were poured in test tubes and left to rest overnight at room temperature to reach equilibrium, before observation between cross-polarizers.

Theoretical Considerations and Comparison with Experimental Results. Following Onsager theory, Vroege and Lekkerkerker ${ }^{20}$ defined the I-N transition by means of a dimensionless constant $c$

$$
c=B_{2}^{\text {iso }} \rho=\frac{\pi}{4} D L^{2} \frac{N}{V}=v_{0} \frac{L}{D} \frac{N}{V}=\frac{L}{D} \Phi
$$

where $B_{2}{ }^{\text {iso }}$ is the second virial coefficient, that is the interaction between two rods and therefore the excluded volume $(\pi / 4) D L^{2}$, and $\rho$ the critical concentration of rods per total volume $V$. With $v_{0}=(\pi / 4) D^{2} L$ as the real volume of a rod, the constant $c$ can be defined simply with the dimensions of the particle and volume fraction $\Phi$.

At the isotropic-nematic transition, the constant reaches a critical value, which is 4 for rigid, uncharged, and monodisperse rods, leading again to eq 1 . For more complex systems, however, the critical value of the constant $c$ changes and is also dependent on the algorithm used to calculate the I-N bifurcation point. ${ }^{20}$

In the case of double layer charges, the excluded volume can be accounted for by an effective excluded volume, $(\pi / 4) D_{\text {eff }} L^{2}$, while the occupied volume remains $(\pi / 4) D^{2} L$. Thus, eq 4 becomes

$$
c=B_{2}^{\text {iso }} \rho=\frac{\pi}{4} D_{\text {eff }} L^{2} \frac{N}{V}=v_{0} \frac{D_{\text {eff }} L}{D^{2}} \frac{N}{V}=\frac{D_{\text {eff }} L}{D^{2}} \Phi
$$

Application of eq 5 to charged semiflexible protein amyloid fibers still requires the change of contour length $L$ into persistence length $L_{\mathrm{p}}$ and the adjustment of the constant $c$ to a value equal to 6 , in agreement with the treatment of Khokhlov and Semenov ${ }^{21}$ and Vroege and Lekkerkerker, ${ }^{20}$ so that the final eq 6 can be used to interpret the critical volume fraction of objects $\Phi_{\text {IN }}$ at the I-N transition in amyloid fibers

$$
\phi_{\mathrm{IN}}=6 \frac{D^{2}}{D_{\mathrm{eff}} L_{\mathrm{P}}}
$$

(20) Vroege, G. J.; Lekkerkerker, H. N. W. Phase transitions in lyotropic colloidal and polymer liquid crystals. Rep. Prog. Phys. 1992, 55, 1241-1309.

(21) Khokhlov, A. R.; Semenov, A. N. Liquid-crystalline ordering in the solution of long persistent chains. Phys. A: Stat. Theor. Phys. 1981, 108, 546-556. 
Table 1. Comparison of Experimental Results with Theoretical Results for the Isotropic-Nematic Phase Transition of $\beta$-Lactoglobulin Fibers at Different pH Values

\begin{tabular}{lcccc}
\hline $\mathrm{pH}$ & overall charge per monomer & effective diameter $(\mathrm{nm})$ & theoretical $\mathrm{I}-\mathrm{N}$ transition $(\%)\left(L_{\mathrm{p}}=1980 \mathrm{~nm}\right)$ & experimental $\mathrm{I}-\mathrm{N}$ transition $(\%)$ \\
\hline 1 & +21 & 20.4 & 0.24 & 0.24 \\
1.5 & +21 & 20.4 & 0.24 & 0.3 \\
2 & +20 & 20.1 & 0.24 & 0.4 \\
2.5 & +19 & 19.8 & 0.26 & 0.4 \\
3 & +16.5 & 18.9 & 0.28 & 0.3 \\
3.5 & +13.1 & 17.5 & 0.31 & 0.3 \\
3.8 & +10 & 15.9 & 0.5
\end{tabular}

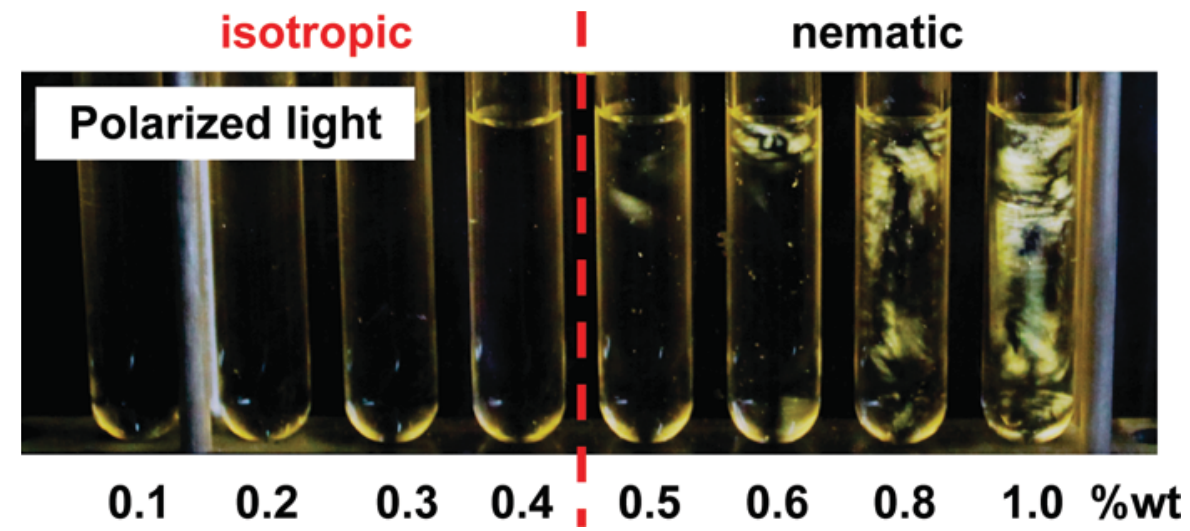

Figure 2. Isotropic-Nematic (I-N) transition observed for $\beta$-lactoglobulin fibers at the ionic strength of $25 \mathrm{mM}$ NaCl. Observation between cross-polarizers.

Table 2. Comparison of Experimental Results with Theoretical Results for the Isotropic-Nematic Phase Transition of $\beta$-Lactoglobulin Rod-Like Aggregates at pH 2 and Different Ionic Strengths

\begin{tabular}{|c|c|c|c|c|c|}
\hline $\begin{array}{l}\text { ionic strength } \\
(\mathrm{mM} \mathrm{NaCl})\end{array}$ & $\begin{array}{l}\text { total ionic strength } \\
(\mathrm{mM})\end{array}$ & $\begin{array}{l}\text { Debye length } \\
(\mathrm{nm})\end{array}$ & $\begin{array}{l}\text { effective diameter } \\
\text { (nm) }\end{array}$ & $\begin{array}{l}\text { theoretical } \mathrm{I}-\mathrm{N} \text { transition } \\
\quad(\%)\left(L_{\mathrm{p}}=1980 \mathrm{~nm}\right)\end{array}$ & $\begin{array}{l}\text { experimental } \mathrm{I}-\mathrm{N} \\
\text { transition }(\%)\end{array}$ \\
\hline $0($ no $\mathrm{NaCl})$ & 10 & 3.0 & 20.1 & 0.24 & 0.4 \\
\hline 1 & 11 & 2.9 & 19.0 & 0.25 & 0.4 \\
\hline 10 & 20 & 2.1 & 13.5 & 0.36 & 0.5 \\
\hline 25 & 35 & 1.6 & 9.7 & 0.50 & 0.4 \\
\hline 50 & 60 & 1.2 & 7.1 & 0.68 & 0.4 \\
\hline 75 & 85 & 1.0 & 5.8 & 0.84 & 0.5 \\
\hline
\end{tabular}

Equation 6, accounting for the double layer effect directly in the excluded volume, has very different behavior than what previously used to predict $\mathrm{I}-\mathrm{N}$ transition in amyloid fibers. ${ }^{15,16}$ In particular, for a fixed persistence length, $\phi_{5} \approx\left(D_{\text {eff }}\right)^{-1}$ as observed for other charged biological systems, ${ }^{5}$ which contrasts with $\phi_{\mathrm{IN}} \approx D_{\text {eff }}$ previously used in charged amyloid protein fibers.

We use eq 6 to study the $\mathrm{I}-\mathrm{N}$ transition as a function of $\mathrm{pH}$ and ionic strength in water dispersions of semiflexible amyloid fibers. Figure 1 shows the experimental vs theoretical value of the $\mathrm{I}-\mathrm{N}$ transition for $\beta$-lactoglobulin fibers, obtained as described in the Experimental Section and as reported in our earlier work. ${ }^{15}$ The $\mathrm{pH}$ has, as a main effect, a change of the linear charge density of the fibers and thus $D_{\text {eff }}$ (see Table 1). The persistence length $L_{\mathrm{p}}$, the diameter $D$, and the Debye length $k^{-1}$ were held fixed in our work to $1980 \mathrm{~nm}, 4 \mathrm{~nm} \mathrm{~nm}$, and $3 \mathrm{~nm}$, respectively. ${ }^{15}$ As can be observed, excellent agreement is found within experimental error between the theoretical predictions (dashed line) and experimental $\mathrm{I}-\mathrm{N}$ transition over the $\mathrm{pH}$ range $1.5-4$.

In order to further explore alternative ways to vary the $\mathrm{I}-\mathrm{N}$ transition by altering $D_{\text {eff }}$, we have maintained constant $\mathrm{pH}$, and thus the linear charge density, while we have altered the "thickness" of the double charge layer by changing the ionic strengthand thus the Debye length - with addition of $\mathrm{NaCl}$. For a specific, fixed ionic strength, $\mathrm{I}-\mathrm{N}$ transition is revealed by concentrating the $\beta$-lactoglobulin fibers until birefringence under cross-polarized

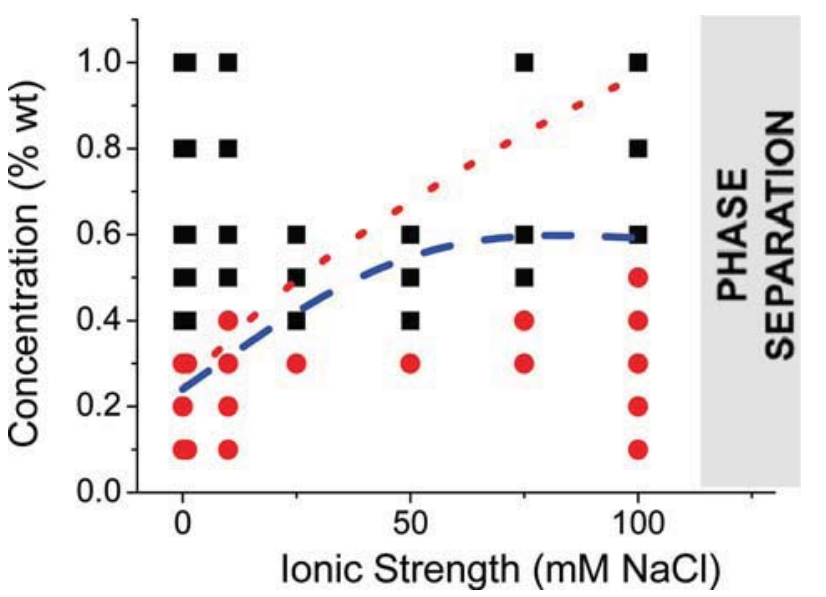

Figure 3. Dependence of the $\beta$-lactoglobulin fiber isotropicnematic $(\mathrm{I}-\mathrm{N})$ transition on ionic strengths at constant $\mathrm{pH} 2$. The red spot represents an isotropic phase; the black square represents a nematic phase. The red dotted line represents the theoretical $\mathrm{I}-\mathrm{N}$ transition as predicted using eqs 6 and 7 . The blue dashed line represents the theoretical $\mathrm{I}-\mathrm{N}$ transition taking into account fiber aggregation at high ionic strength.

microscopes appears. Figure 2 shows an iso-ionic strength series used to determine the $\mathrm{I}-\mathrm{N}$ transition at a constant $I$. 

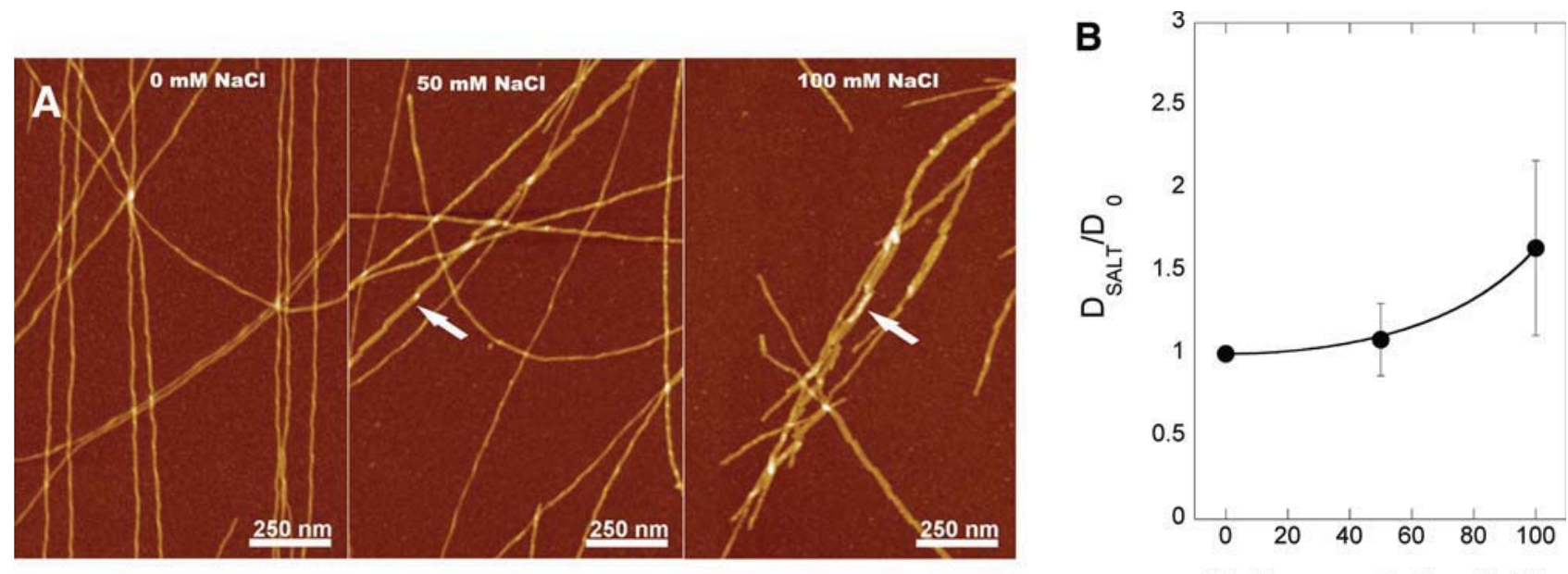

$\mathrm{NaCl}$ concentration (mM)

Figure 4. (A) AFM images of $\beta$-lactoglobulin fibers at different concentration of $\mathrm{NaCl}$. (B) Ratio of the average diameter $D_{\mathrm{SALT}}$ measured at various ionic strengths normalized with respect to the diameter measured at $0 \mathrm{NaCl}$ content $\left(D_{0}\right)$. The continuous line is the interpolation given by $1 /\left(1+b x^{2}\right)$, with $b=-3.894 \times 10^{-5}$. The effective diameter accounting for both charge double layer and aggregation is then found as $D_{\text {eff }}^{\text {agg }}=a \cdot D_{\text {eff }}$ with $a$ being the multiplying factor shown in Figure $4 \mathrm{~b}$.

Variations of the Debye length with ionic strength can be accounted by (see Table 1)

$$
k^{-1}=\sqrt{\frac{\varepsilon_{0} \varepsilon_{\mathrm{r}} k T}{2 N_{\mathrm{A}} e^{2} I}}
$$

where $\varepsilon_{0}$ is the dielectric permittivity in vacuum $\left(\varepsilon_{0}=8.85 \times 10^{-12}\right.$ $\mathrm{F} / \mathrm{m}), \varepsilon_{\mathrm{r}}$ dielectric permittivity in water $\left(\varepsilon_{\mathrm{r}}=80.1\right), k$ Boltzmann's constant $\left(k=1.38 \times 10^{-23} \mathrm{~J} / \mathrm{K}\right), T$ absolute temperature $(293 \mathrm{~K})$, $N_{\mathrm{A}}$ Avogadro's constant $\left(N_{\mathrm{A}}=6.02 \times 10^{23}\right)$, e elementary charge $\left(e=1.6 \times 10^{-19} \mathrm{C}\right)$, and $I$ total ionic strength $\left(\right.$ in $\left.\mathrm{mol} / \mathrm{m}^{3}\right)$. When $\mathrm{NaCl}$ is not present, using eq 7 and the ionic strength of pure water at $\mathrm{pH} 2(10 \mathrm{mM})$, one again finds $3.0 \mathrm{~nm}$ for the Debye length. By calculating the variation of Debye length and the effective diameter on ionic strength (eqs 2, 3, and 7), the critical concentration at which the nematic phase appears can again be calculated using eq 6 (see Table 2). Figure 3 shows the concentrations at which the nematic phase appears at $\mathrm{pH} 2$ and the various ionic strengths explored. The symbols locate the experimental I-N transitions, whereas the red dotted line gives the $\mathrm{I}-\mathrm{N}$ transition as predicted by eq 6 . At low ionic strengths, an excellent agreement is found again, whereas the deviation between experimental data and theoretical predictions rises with increasing ionic strength.

For our system, rationalizing the deviation between theoretical and experimental values at increasing ionic strength is nontrivial. Indeed, none of the three most straightforward modifications of the Onsager theory, e.g., Odijk twisting parameter, ${ }^{17}$ variable persistence length with ionic strength, ${ }^{22}$ and counterion condensation, ${ }^{23,24}$ can explain such a divergence.

The Odijk twisting parameter, defined as $h=k^{-1} / D_{\text {eff, takes }}$ into account the lowering of energy that two aligned rod-like charged objects can gain by rotating with respect to each other by $90^{\circ} .{ }^{17}$ In the present case, $h$ is fairly small $(h<0.18$; see Table 2$)$, and thus, the estimated $\mathrm{I}-\mathrm{N}$ compositions should be affected by less than $15 \%$ by inclusion of the twisting effect in the energy virial expansion; ${ }^{17}$ furthermore, for semiflexible objects, this effect should be even smaller. Yet, when including $h$ in the virial expansion of the free energy, a small delay of the onset of the I-N transition is to be expected, which is opposite to the trend

(22) Aymard, P.; Nicolai, T.; Durand, D.; Clark, A. H. Static and dynamic scattering of $\beta$-lactoglobulin aggregates formed after heat-induced denaturation at pH2. Macromolecules 1999, 32, 2542-2552.

(23) Deserno, M.; Holm, C.; May, S. Deserno. Macromolecules 2000, 33, 199 206.

(24) Dobrynin, A. V.; Rubinstein, M. Theory of polyelectrolytes in solutions and at surfaces. Prog. Polym. Sci. 2005, 30, 1049-1118. observed in Figure 3 when comparing the red dotted line and experiments.

With respect to the persistence length, Aymard et al. ${ }^{22}$ have shown that for $\beta$-lactoglobulin fibers made at low ionic strength, this decreases slowly with increasing ionic strength compared to other classical polyelectrolytes and suggested that other factors contribute to the rigidity of protein fibers, such as hydrogen bonding $\beta$-sheets. Nonetheless, even if a decreasing persistence length of the $\beta$-lactoglobulin fibers with increasing ionic strength is considered in our work, the theoretical $\mathrm{I}-\mathrm{N}$ transition is expected to further increase with respect to the red dotted line prediction, according to eq 6 . This would again lead to an even larger departure between experimental data and theoretical predictions.

Finally, counterion condensation cannot be invoked, to account for the disagreement found in Figure 3. Counterion condensation is predicted to occur when the Oosawa-Manning parameter $\left(v_{\mathrm{eff}} \cdot Q\right) / e>1,{ }^{24}$ and recent theoretical work has shown that this equation also holds when extra salt is added. ${ }^{23}$ In the case of the present study, by taking $v_{\text {eff }} \approx 5 e / \mathrm{nm}$ (see Table 1 ) and $Q=0.7 \mathrm{~nm},{ }^{15}$ one finds that the Oosawa-Manning criterion $5 \cdot 0.7>1$ is met and thus that counterion condensation is likely to occur, leading to a decrease of both the ionic strength and linear charge density $v_{\text {eff. }}$. A quick evaluation of the maximum amount of condensable counterions can immediately be performed to rule out strong effects on the ionic strength: considering the low fiber concentration and the molar concentrations of salt used, even in the event of massive counterion condensation leading to complete neutralization of the charges on the fibers, the ionic strength would be essentially unaffected ( $<5 \%$ variation), e.g., any possible reduction in ionic strength is too small to explain the trends of the Figure 3. On the other hand, in the double layer picture of the Onsager theory, $D_{\text {eff }}$ would be greatly decreased by the reduction of $v_{\text {eff }}$ (see eqs 3 and 2), and thus, $\phi_{\text {IN }}$ increased, leading again to an enhanced deviation between experimental data and theoretical estimations found in Figure 3 (red dotted line).

Thus, the sources of discrepancy at increasing ionic strength between theory and experimental data emerging from Figure 3 need to be found in other factors.

Very recently, aggregation among protein amyloid fibers has been shown to occur based on the interplay between long-range electrostatic repulsive forces and short-range hydrophobic interactions. ${ }^{25}$

(25) Adamcik, J.; Jung, J.-M.; Flakowski, J.; De Los Rios, P.; Dietler, G.; Mezzenga, R. Understanding Amyloid Aggregation by Statistical Analysis of Atomic Force Microscopy Images. Nat. Nanotechnol. 2010, doi:10.1038/ nnano. 2010.59 
Increasing ionic strength is expected to further enhance aggregation. Figure 4a shows the AFM images of fiber bundles observed at increasing ionic strength. As can be observed, increasing ionic strength results in an increasing aggregation tendency and increasing average diameter of the aggregated fibers. Figure $4 \mathrm{~b}$ shows the evolution of the average diameter measured (AFM width) normalized with respect to the diameter of the fibers at zero salt content. Because aggregation does not alter the volume fraction of the fibers ( $\phi$ in eq 5), the effect of aggregation can be accounted for in the Onsager treatment directly by adjusting $D_{\text {eff }}$ to account for this effect. This is essentially achieved by rescaling $D_{\text {eff }}$ into a new $D_{\text {eff }}^{\text {agg }}=a \cdot D_{\text {eff }}$ in which $a$ is the prefactor shown in Figure $4 \mathrm{~b}$ embodying the aggregation among fibers.

The use of eq 6 in which $D_{\text {eff }}$ is replaced by $D_{\text {eff }}^{\text {agg }}$ to interpret the I-N transition at increasing ionic strengths is shown by the dashed blue line in Figure 3. By performing this correction, a satisfactory agreement is found in this case between experiments and theoretical estimations over the full ionic strength window investigated. Above $125 \mathrm{mM}$, the aggregation becomes so strong that the solution is not transparent anymore and macroscopic phase separation occurs.

Therefore, in the present system Onsager theory is not applicable for ionic strengths higher than $125 \mathrm{mM}$.
The present findings clearly show that if the double layer caused by charges and counterions is accounted for directly in the excluded volume and if the aggregation of fibers occurring at high ionic strength is correctly implemented in the treatment, Onsager theory is suitable to describe in an accurate quantitative way the $\mathrm{I}-\mathrm{N}$ transition onset in charged semiflexible protein amyloid fibers. This also implies that obtaining concentrations for $\mathrm{I}-\mathrm{N}$ transitions using the simplified $\Phi_{\mathrm{IN}} \approx D_{\text {eff }} /$ $L_{\mathrm{p}}$ criterion is an oversimplification, which requires rescaling of critical concentrations by a $\left(D / D_{\text {eff }}\right)^{2}$ correction factor and that $D_{\text {eff }}$ must account for the increase arising from both the charge double layer and fiber aggregation. We finally observe that, despite the encouraging agreement between the experimental findings and the onset of the isotropic-nematic transition predicted theoretically by eq 6 , no coexistence of $\mathrm{I}$ and $\mathrm{N}$ is found in this work, whereas eq 1 and eq 6 refer more exactly to a bifurcation point, that is, to the onset of coexistence of the isotropic and nematic phases. This discrepancy is analogue to that observed by Fraden et al. ${ }^{5}$ in other biological rod-like objects (tobacco mosaic virus), and the physical reasons behind this experimental evidence remain obscure up to the present time. 